\title{
LITERARY, POLITICAL AND CULTURAL STAKES IN THE TEXTBOOKS OF FRENCH -KALEIDOCOPE, ECRITURE, BEAUBOURG AND RIVE GAUCHE EXAMPLE: THE LAST YEAR OF THE ITALIAN HIGH SCHOOL
}

\author{
[ENJEUX LITTERAIRES, POLITIQUES ET CULTURELS DES \\ MANUELS DE FRANÇAIS: KALEIDOCOPE, ECRITURES, \\ BEAUBOURG ET RIVE GAUCHE \\ EXEMPLE : DERNIERE ANNEE DU LYCEE ITALIEN]
}

\author{
Cynthia Etheves
}

\section{doi: 10.18355/PG.2016.5.1.29-49}

\begin{abstract}
In the Italian high school textbooks of the previous year there is no point "lexicon" or "grammar". The studied lexicon is the one met during the extracts of the literary texts. What are then the arguments treated by these textbooks? What are the didactic, literary, political and cultural stakes? What are choices made by the authors of these textbooks?
\end{abstract}

\section{Keywords}

textbooks, Italy, literature, culture, politics

\section{Résumé}

Dans les manuels de dernière année du lycée italien il n'y a pas de point « lexique » ou de point « grammaire ». Le lexique étudié est celui rencontré lors des extraits des textes littéraires. Quels sont alors les arguments traités par ces manuels ? Quels sont les enjeux didactiques, littéraires, politiques et culturels ? Quels sont les choix faits par les auteurs de ces manuels scolaires?

\section{Mots Clés}

manuels scolaires, Italie, littérature, culture, politique

\section{Le cadre littéraire dans les manuels analysés}

Nous allons dans un premier temps comparer Kaléidoscope avec Ecritures. Puis nous allons faire le même travail avec Beaubourg et Rive Gauche afin de comparer les méthodes des deux maisons d'édition.

Dans Kaléidoscope, on peut avoir un sentiment de désordre par rapport à la rigueur d'Ecritures. En effet, Vigny se trouve dans les rubriques poésie romantique, théâtre romantique et courant européen. Musset trouve sa place dans la poésie mais aussi le théâtre. Et Hugo, quand à lui, se retrouve dans les catégories : théâtre, Hugo et roman européen.

Ce désordre est dû au fait que, contrairement à Ecritures, Kaléidoscope classe ses unités en fonction des genres littéraires et non des auteurs. Ceci nous autorise à dire que pour le manuel Ecritures, les auteurs sont plus 
importants que les genres littéraires. Les auteurs présents dans les deux manuels dont le nom figure dans le titre de l'unité sont au nombre de douze : Chateaubriand, Hugo, Balzac, Stendhal, Flaubert, Zola et Maupassant, Baudelaire, Verlaine et Rimbaud, Proust, Gide, Céline puis Camus. Certains auteurs sont plus importants pour Ecritures puisque leur nom figure dans le titre de l'unité alors dans Kaléidoscope, ces auteurs sont nommés dans l'étape de l'unité : Lamartine et Vigny, Musset et Nerval, Le conte de L'Isle, Apollinaire, Sartre et Mme de Staël. D'autres, en revanche semblent plus importants dans Kaléidoscope : Zola possède une unité à lui tout seul, intitulé Germinal comme son roman. Marguerite Yourcenar a son nom qui figure dans le titre de l'unité. D'autres encore ne sont nommés qu'une seule fois dans le manuel de Kaléidoscope comme Nerval, Mme de Staël, Senancour, Constant, Mauriac, Breton, Aragon, Eluard, Giono, Malraux, Saint Exupéry et Vian, alors qu'Ecritures réserve à certains d'entre eux une unité.

Si l'on compare Beaubourg et Rive Gauche, on note que Beaubourg possède des auteurs absents de Rive gauche : Benjamin Constant (25 vol A), Prosper Mérimée (119 vol. A), Edmond et Jules de Goncourt (203 vol. A), Jules Barbey d'Aurevilly (273 vol. A), Edmond Rostand (283 vol. A), Paul Valéry (19 vol. B), Paul Claudel (86 vol. B), Nathalie Sarraute (197 vol. B), Alain Robbe-Grillet (202 vol. B), et Albert Cohen (237 vol. B). On rappelle le but de Rive Gauche de faire « una cura dimagrante » et d'être plus « snello » que son ainé. Ceci justifie les absences de certains auteurs dans Rive Gauche.

\section{Le choix des auteurs dans les manuels analysés}

Le choix des auteurs ne semble pas être anodin. Commençons par en observer quelques-uns. Dans Ecritures, Chateaubriand, est «nommé secrétaire d'Ambassade à Rome » en 1803, et rédige Les Voyages en Italie (1826). Il écrit aussi un «véritable pamphlet contre Napoléon ». On souligne son lien avec l'Italie et son hostilité envers Napoléon. Hostilité reprise par Hugo mais aussi par Madame de Staël qui « est condamnée à l'exil pendant dix ans à cause de son opposition à Napoléon ». Durant ces dix années, elle effectue elle aussi un voyage en Italie. Des poètes tels que José Maria de Heredia "évoquent les pays lointains, la Grèce et la Sicile, Rome et les Barbares », Lamartine, quant à lui, dès lors de son premier voyage en Italie, "s'éprend d'une jeune Napolitaine ». Toujours dans Ecritures, Zola est «le fils d'Italien» et Stendhal est défini comme un italien et non comme un français. En effet, pour ce dernier, "L'Italie occupe une place très importante. Il la découvre tout jeune, à 17 ans, à la suite de Napoléon, participant à la campagne d'Italie. En 1821, la France perd l'Italie. Stendhal cherchera à y séjourner le plus possible en tant que diplomate. Cet amour passionné l'accompagnera toujours : il aime l'atmosphère de la péninsule, ses paysages, ses villes, ses habitants, ses femmes. C'est pour lui le pays de la beauté, le plaisir de vivre et de l'amour. On l'appellera «l'Italien » et il préparera lui-même son épitaphe : Henry Beyle, le Milanais. » Voici un bel éloge à l'Italie. 
Ces mêmes auteurs se retrouvent dans Kaléidoscope qui souligne «la carrière littéraire et l'hostilité à Napoléon» de Chateaubriand qui a «été ambassadeur à Rome». Stendhal «entre dans Milan sur les pas de Bonaparte. L'Italie est une révélation pour lui », « et surtout il goute l'Italie qui le séduit de plus en plus ». Et Zola est « le fils d'italien », « le fils d'un ingénieur italien naturalisé ».

Dans Rive Gauche, Lamartine « entreprend une carrière diplomatique qui le conduira d'abord à Naples, puis à Florence », Flaubert « voyage au Moyen Orient, en Grèce, en Italie » et Stendhal « est sous-lieutenant dans l'armée d'Italie, un pays qui le touche profondément - « Cinq à six mois de bonheur céleste, complet », dira-t-il de cette période ».

\section{Choix des extraits littéraires dans les manuels analysés}

Quels sont les extraits littéraires choisis par les auteurs des manuels que nous analysons ? Sont-ils identiques ? Ou bien varient-il d'un manuel à un autre. Observons Kaléidoscope et Ecritures, puis Beaubourg et Rive Gauche afin de mieux comparer les deux maisons d'édition. Les extraits littéraires de Kaléidoscope et d'Ecritures sont en général différents. Quelques extraits sont identiques comme Le Spleen de Baudelaire, Il pleut d'Apollinaire ou encore "Correspondance secrète dans La Chartreuse de Parme de Stendhal ». D'autres sont un peu plus courts dans Ecritures que dans Kaléidoscope comme par exemple « René de Chateaubriand» ou bien «J'accuse » de Zola.

Les extraits littéraires de Beaubourg sont en général identiques à ceux de Rive Gauche. Cependant, Beaubourg possède des extraits littéraires supplémentaires par rapport à Rive Gauche. Ce seront par exemple, Le Lys dans la vallée de Balzac, Les Contemplations de Hugo, L'Education sentimentale de Flaubert ou encore Nana ou Lamiel de Zola. On se rappelle alors le but de Rive Gauche de faire « una cura dimagrante » et d'être plus «snello » que son ainé. Ceci justifie l'absence de certains extraits littéraires dans Rive Gauche.

Ecritures essaie d'être plus innovatrice et plus moderne que son ainé alors que Rive Gauche est un "concentré » de son ainé. On peut parler ici de deux catégories. La première est composée des textes incontournables tels que Notre Dame de Paris ou bien Les Misérables de V. Hugo, Germinal de Zola, Mme Bovary de Flaubert ou encore L'Etranger et La Peste de Camus. Mais sont-ils les mêmes pour chaque manuel? Pour répondre à cette question, nous avons repris les auteurs les plus cités et nous avons relevé les œuvres présentes dans les quatre manuels. Voici les œuvres que nous avons relevé : les incontournables de Chateaubriand sont René et les Mémoires d'Outres Tombe; de Victor Hugo les contemplations, les Orientales, Hernani et les Misérables; de Balzac, Eugénie Grandet et Le Père Goriot, de Stendhal Le Rouge et le noir, la Chartreuse de Parme ; de Flaubert on retrouve Mme Bovary et l'Education sentimentale. Pour Zola nous avons vu L'Assommoir et Germinal ; pour Baudelaire Le Spleen et pour Camus La Peste et L'Etranger.

Slavonic Pedagogical Studies Journal, ISSN 1339-866o, Volume 5 Issue 1, February 2016 
La deuxième catégorie se compose d'extraits liés à l'Italie comme Lorenzaccio et Les Caprices de Marianne de Musset, La Chartreuse de Parme de Stendhal, Les Caves du Vatican de Gide ou encore le Temps retrouvé de Proust. Si on approfondit cette analyse on note que le texte de Proust est intitulé dans Ecritures «C'était Venise » et que l'extrait ne parle que de Venise. De même l'extrait de la chartreuse de Parme est intitulé «Milan en 1796 », toujours dans Ecritures. Reprenons les textes que les quatre manuels possèdent en commun: Lorenzaccio, La Chartreuse de Parme, Les Caves du Vatican. Nous voulons souligner qu'Ecritures possède plus d'extraits littéraires parlant de l'Italie. On peut parler par exemple de Voyage en Italie de Chateaubriand, Corinne de Mme de Staël (Corinne vit à Rome en Italie) ou encore Vanina Vanini de Stendhal (l'un des huit récits des Chroniques Italiennes, posth.1855).

\section{Le lien avec l'Italie dans les manuels analysés}

Nous avons vu jusqu'à présent qu'une grande partie des auteurs étudiés ont un vécu italien et que ce vécu se retrouve dans certains des extraits proposés par les manuels. D'autres auteurs ou d'autres extraits littéraires n'ont pas de lien particulier avec l'Italie, cependant ils sont inclus dans les manuels car ils permettent de créer un «trait d'union » entre la France et l'Italie comme précise le Kaléidoscope. Ce «trait d'union» s'intitule « liaisons » chez Ecritures, «d'un pays à l'autre » et «d'un siècle à l'autre » chez Beaubourg et Rive Gauche. Quelle partie de l'histoire littéraire est mise en valeur? Pourquoi?

\section{Romantisme français ou romantisme italien dans les manuels analysés}

Un lien unit les auteurs du romantisme: l'Italie et/ou l'hostilité envers Napoléon. Nombreux sont les auteurs ayant séjourné en Italie pour une raison ou une autre (Chateaubriand est nommé secrétaire d'Ambassade à Rome en 1803, Lamartine, lors d'un de ses voyages s'éprend d'une jeune Napolitaine : Graziella ou encore le couple Stand et Musset qui mettent fin à leur amour à Venise...). Parmi ces auteurs, il y a aussi Madame de Staël, qui propose de «traduire les chefs-d'œuvres littéraires dans les différentes langues européennes afin de donner des modèles aux écrivains de chaque nation, en particulier aux italiens qui se sont retranchés dans une admiration stérile des classiques, et leur permettre ainsi de s'inspirer des littératures étrangères $\gg$ Rive Gauche, 16).

D'autres ont un lien commun avec les auteurs des manuels : une hostilité envers les Napoléon. Hugo par exemple dénonce Napoléon dans Les Châtiments / Cette hostilité est partagée par Madame de Staël et son amant Benjamin Constant. Balzac, en revanche, est considéré comme un copieur. De par sa Comédie Humaine, il «est ambitieux parce qu'il fait explicitement référence à Dante et à sa Divine Comédie mais en inversant les priorités : c'est l'homme qui est au centre de l'édifice Balzacien. » (78 d'Ecritures et 70 de Kaléidoscope). Stendhal est directement appelé «l'italien» et se déclare lui-même dans son épitaphe «Henry Beyle, le Milanais ». 
Ces auteurs qui ont vécu une période de leur vie en Italie décrivent à travers leurs œuvres l'Italie, ou mettent en scènes des personnages italiens. Pour Stendhal, l'Italie est le «pays de la beauté, du plaisir de vivre et de l'amour ». Dans Rome, Naples et Florence, il raconte l'Italie et « fait sentir la richesse culturelle de ce pays ». Une note d'orgueil national se faufile à travers ces commentaires. C'est pourquoi grand nombres d'extraits littéraires ont un lien avec l'Italie, par exemple Corinne ou l'Italie de Madame de Staël, ou encore Lorenzaccio (1834) de Musset qui retrace un fait historique : l'assassinat en 1537 du Duc de Médicis par Lorenzo de Médicis à Florence.

L'ère romantique s'achève par une double page de «liaisons» dans Ecritures, par un dossier dans Kaléidoscope sur les héros romantiques: Nous distinguons, dans ce document deux grandes parties. La première partie traite du romantisme au niveau Européen. Seules dix-sept lignes lui sont consacrées. La deuxième partie, plus volumineuse, comporte vingtneuf lignes, et est réservée au romantisme italien, ou plus exactement, à la comparaison entre romantisme français et romantisme italien. Toutefois dans la première partie, il aussi question des auteurs romantiques italiens. Nous comprenons pourquoi le système littéraire français est comparé au système littéraire italien : ces manuels sont écrits pour un public italien. Quels sont les aspects littéraires mis en relief par cet exemple? Dans l'extrait ci-dessus, l'influence des auteurs italiens par Madame de Staël est clairement soulignée (Giovanni Berchet et Alessandro Manzoni). Mme de Staël publie un article sur La manière de traduire et l'utilité des traductions. Dans ce même exemple, nous sont aussi présentés les points de vue de Berchet et Manzoni sur le romantisme. Nous retrouvons ici la même approche que dans le manuel Kaléidoscope : elle est purement théorique. Voici comment les manuels Beaubourg et Rive Gauche explique l'influence de Mme de Staël : Mme de Staël «suggère de traduire les chefs d'œuvres littéraires dans les différentes langues européennes afin de donner des modèles aux écrivains de chaque nation, en particulier aux Italiens qui se sont retranchés dans une admiration stérile des classiques, et leur permettre ainsi de s'inspirer des littératures étrangères ». Ce que propose en fait ici Madame de Staël, c'est de prendre la littérature française comme modèle. À ces mots, Giacomo Léopardi répond « ebbene date dunque agl'Italiani altri modelli », il demande un autre modèle que le modèle français. Il considère le conseil de Mme de Staël comme «vanissimo », vaniteux. Nous pouvons par ailleurs relever ici, l'orgueil italien face à la littérature française.

Observons à présent la ressemblance des héros romantiques, par exemple entre Jacopo Ortis (1802) de Foscolo et René (1802) de Chateaubriand. « Il s'agit de personnages dominés par la mélancolie, l'inquiétude, l'angoisse, le refus de la réalité » (Kaleidoscope), ce sont des personnages tristes qui souffrent du « mal du siècle ». Dans Kaléidoscope, cet aspect est traité dans le dossier «Le héros romantique », bien qu'il ne soit pas approfondi comme dans Ecritures, où l'argument est illustré au travers d'extraits littéraires, tout comme dans une unité normale du manuel. Cependant, observons le «trait d'union ». 
Le premier texte est extrait de Les souffrances du jeune Werther de Goethe (1774) traduit en italien. C'est en langue maternelle italienne que l'extrait est proposé aux apprenants italiens. Il exprime «le mal de vivre, l'amour malheureux, la tentation du suicide ». Cette phrase qui explique le texte est en français.

Le deuxième texte est extrait de Zibaldone de Leopardi, c'est un extrait de la littérature italienne, il reste donc en langue italienne.

Observons de plus la page 102 qui est «L'entrée en matière » du dossier : Cette page d'introduction, donne comme exemple quatorze héros romantiques qui vont être traités durant le dossier ou bien qui ont été vus dans les unités précédentes. Nous retrouvons les mêmes exemples que dans le manuel Ecritures: René (Chateaubriand), Oberman (Senancour), Adolphe (Constant). Kaléidoscope y ajoute Ruy Blas (Hugo), Hernani (Hugo) et Emma Bovary (Flaubert).

Comment est traité le thème des héros romantiques dans Beaubourg et Rive Gauche? Une rubrique «Au premier plan» présente ce thème. Elle parle $\mathrm{du}$ « héros romantique » et du «mal du siècle». Qu'est-ce que le héros romantique selon ces manuels?

Le héros romantique est «jeune» et «s'ennuie», il est extrêmement sensible et sa «sensibilité » le porte à «s'isoler pour admirer des paysages ", il contemple. Les manuels restent purement théoriques. Qu'estce que le mal du siècle selon les manuels étudiés ? Après avoir donné l'extrait de Chateaubriand qui explique ce «mal » à sa façon, il nous est dit qu'il s'agit d'un «mal moderne, sans illusions et pourtant débordant d'énergie », ce que nous appellerions aujourd'hui la dépression.

Un autre point est traité dans cette liaison, il s'agit de la dimension chrétienne de Chateaubriand et de Manzoni. En effet, dans l'extrait de René d'Ecritures page 23 «O Dieu! Si tu, m'avais donné une femme selon mes désirs ; si comme notre premier père, tu m'eusses amené par la main une Eve tirée de moi-même... » Chateaubriand fait référence à la bible et à l'apparition de la femme sur la terre dans la genèse.

Kaléidoscope reprend l'aspect religieux dans ces quelques lignes (31):

«Atala, Les Martyres, le Génie du christianisme sont des ouvres où Chateaubriand exprime ses profondes convictions chrétiennes, spirituelles et morales. »

Ainsi, le «précurseur » du romantisme a été influencé par la religion et ces œuvres en témoignent.

Puis, toujours dans Kaléidoscope, on nous confirme que le romantisme est une période profondément marquée par le christianisme (44) :

«Toujours après le culte de la raison, le romantisme revient à une dimension spirituelle et aux sources du christianisme. Mais parallèlement, il manifeste aussi un attrait pour les mystérieuses forces du mal. »

Les héros luttent donc contre des «forces du mal» ce pourquoi beaucoup cherchent à « se réfugier dans la religion ».

Dans Beaubourg (13) et dans Rive Gauche, nous pouvons lire une partie sur la ferveur religieuse du $\mathrm{XIX}^{\circ}$ siècle :

Dans cet extrait nous comprenons que, à l'ère romantique, le peuple peut schématiquement être divisé en « traditionalistes », contre la séparation des 
pouvoirs de l'Eglise et de l'Etat, d'une part, et en « libéraux », « favorables à la séparation de l'Eglise et de l'Etat », d'autre part. Cette division peut se retrouver parmi les auteurs, par exemple Chateaubriand, Mme de Staël sont liés au christianisme à la différence de Hugo ou de Sand. Si Beaubourg et Rive Gauche relèvent clairement ces deux parties, c'est pour souligner l'importance de la question de la séparation des pouvoirs, qui se pose à l'époque. En effet, l'Italie est un pays où le christianisme a une forte influence, et il n'y a pas été question de division entre pouvoirs étatique et pouvoir ecclésiastique. Ceci est surement dû, entre autre, au fait que le Vatican se trouve sur le territoire italien. Nous avons peut être ici une des clés de lecture des manuels Kaléidoscope et Ecritures, ceux-ci mettent en effet l'accent uniquement sur l'aspect positif du christianisme, et sur les auteurs qui sont fortement influencés par le christianisme.

Passons désormais au lien entre Lamartine, Vigny et Leopardi, traités dans chacun de nos manuels. Les trois auteurs ont en commun plusieurs thèmes comme le pessimisme, le désir, l'amour-haine.... Kaléidoscope met en évidence dans sa rubrique «Trait d'union» le lien entre Lamartine et Leopardi de la manière suivante :

Le document se présente en deux colonnes : dans la colonne de droite, un extrait de poésie de Lamartine écrit en langue française, dans la colonne de gauche un extrait de poésie de Leopardi en langue italienne. Les deux auteurs parlent de la condition humaine, des souffrances de l'homme, de son existence et de son utilité sur la terre. Il n'y a aucune explication des deux textes, mais nous comprenons que les thèmes sont identiques, notamment en observant le vocabulaire employé : la peine, la souffrance, le désespoir, la nécessité et la naissance, la mort...etc. Dans Ecritures, il y a deux comparaisons. La première s'effectue, tout comme dans Kaléidoscope entre Lamartine et Leopardie. Cependant, aucun extrait de poèmes n'illustre la comparaison cette fois-ci. Les thèmes semblables d'un poète à un autre sont la «tension vers l'infini », le " conflit rêve-réalité », le "pessimisme historique », le « cosmique », le « malaise existentiel », le « désir de mort », l' « amour-haine pour la nature » et l' « inspiration autobiographique ». Suivent des comparaisons entre différentes œuvres, comme Canti de Leopardi et les Premières Méditations poétiques de Lamartine. On nous propose de comparer plusieurs poèmes: l'Homme, le Désespoir, et la Foi de Lamartine avec l'Ultimo canto di Saffo, A Silvia, Le ricordanze, Canto notturno d'un pastore errante dell'Asia, Dialogo della natura e di un Islandese. Tout d'abord notons que les titres italiens n'ont pas été traduits en français. Ensuite, nous constatons qu'une connaissance préalable des auteurs et de leurs poésies, est indispensable pour établir un lien entre eux. La rubrique «Liaison" peut donc être un moyen de faire une étude comparative en classe sur l'un des poèmes proposés.

En revanche, la deuxième comparaison s'effectue entre Vigny et Leopardi : Tout comme dans Kaléidoscope, la présentation se fait sous la forme de deux colonnes, conduisant ainsi instinctivement l'apprenant à la comparaison. Dans la colonne de droite il y a un extrait de poésie de Vigny

Slavonic Pedagogical Studies Journal, ISSN 1339-866o, Volume 5 Issue 1, February 2016 
écrit en langue française, dans la colonne de gauche un extrait de poésie de Leopardi en langue italienne.

Contrairement à son ainé Kaléidoscope, Ecritures possède un paragraphe d'explication précède les extraits littéraires. On nous y explique l'existence d' « analogies (...) frappantes entre Leopardi et Vigny ». Quels sont ces analogies? Sont cités notamment la «douleur cosmique » et le « pessimisme». Suivent des exemples d'œuvres qui peuvent être comparées comme la Mort du Loup de Vigny et le Dialogo di Plotino e di Porfirio de Leopardi, ou encore le Mont des Oliviers avec le Canto notturno d'un pastore errante dell'Asia et l'Ultimo canto di Saffo, mais aussi la Maison du berger et la Ginestra. Les thèmes semblables sont alors le « sens de la vie », le « silence d'un Dieu », « le problème du bien et du mal », le « mythe de la nature » et la « douleur humaine ». Pour pouvoir faire de telles comparaisons il est nécessaire de connaitre les poésies de Leopardi et de Vigny, ou du moins de les avoirs sous les yeux. Cette liaison proposée entre les deux auteurs peut ainsi être une proposition d'étude à affronter en classe avec les apprenants italiens, ou encore une étude suggérée aux apprenants désireux d'approfondir leur connaissance du romantisme. Nous trouvons finalement une explicitation du lien entre ces deux poésies. Il s'agit de la «nature cruelle pour laquelle la vie humaine n'a pas plus de valeur que celle des fourmis ». Nous rencontrons le thème récurrent des souffrances de l'Homme. Puis, sous les deux colonnes de citations, se trouvent deux autres lignes d'explications: « les poètes invitent (...) leurs semblables à la fraternité ». Le manuel propose quelques vers supplémentaires de Vigny adressés à Eva, «la femme aimée » et aux humains, pour faciliter la recherche «des correspondances et des similitudes avec la Ginestra ».

Tout comme Kaléidoscope, Beaubourg et Rive Gauche proposent une seule comparaison, mais ils choisissent celle entre Vigny et Leopardi.

Les premières lignes sont extraites de La Maison du Berger de Vigny. Au travers de ce poème sont expliqués les liens et les thèmes semblables entre les deux auteurs, tels que la « conception de la nature ». Les dernières lignes du document sont extraites de Dialogo della natura e di un Islandese de Leopardi. L'extrait de Vigny est en langue française et l'extrait de Leopardi est en langue italienne.

Si Kaléidoscope donne seulement des extraits à comparer sans aucun commentaire, les explications de Rive gauche et de Beaubourg sont, elles, minimes. Seul Ecritures propose deux comparaisons et explique clairement le lien entre les auteurs. La rubrique «Trait d'union » de Kaléidoscope peut être vue comme une comparaison entre deux extraits, à effectuer en classe avec les apprenants italiens. La «Liaison» d'Ecriture est un approfondissement et « une proposition d'étude à effectuer. " D'un pays à un autre » de Beaubourg et de Rive Gauche semble n'être qu'un approfondissement.

Le dernier point que propose d'étudier Ecritures porte sur les différences qui peuvent exister entre le romantisme Italien et le romantisme français. Le manuel aborde en premier lieu le romantisme italien : 
«En Italie, la littérature est au service de la construction d'une identité nationale par rapport à l'occupant étranger (...). En Italie, le romantisme coïncide avec le Risorgimento et la poésie italienne de l'époque est surtout une poésie patriotique, faite pour inciter à la lutte, pour exalter l'histoire du passé, pour s'opposer à l'oppression étrangère, pour proposer les valeurs de l'amour pour la patrie, de la liberté et de la fraternité ».

La France à cette période n'a "pas d'occupant et l'unité est déjà faite depuis longtemps ». De la sorte les auteurs des manuels d'Ecritures minorisent le rôle unificateur du romantisme français, pourtant postrévolutionnaire, et accentuent l'importance de son rôle en Italie lors du Risorgimento. Seul Ecritures propose d'analyser la différence entre le romantisme italien et le romantisme français. Le manuel ajoute quelques lignes d'explications sur le développement du romantisme au nord de l'Italie. Après avoir spécifié la ville où le romantisme se développe principalement en Italie :

«C'est surtout à Milan et dans le nord d'Italie que le mouvement romantique se développe »

On nous donne une explication à ce phénomène : «Le Nord était en effet plus ouvert aux influences étrangères parce que les écrivains comme Staël, Byron, Stendhal y avaient séjourné ». C'est donc l'influence des écrivains français venant en Italie qui permet au romantisme de se développer dans le nord d'Italie.

\section{Naturalisme français ou vérisme italien dans les manuels analysés}

En ce qui concerne le lien entre le naturalisme français et le vérisme italien, nous notons que l'espace qui y est consacré est plus étroit que celui consacré au romantisme. Dans la rubrique «Trait d'union» de Kaléidoscope, nous relevons deux petits textes explicatifs. Le premier texte compare «la bohème parisienne » et la «scapigliatura» italienne. Les bohémiens sont «des artistes » qui sont «en marge de la société » tout comme les tziganes que l'on disait de Bohème. C'est 1'écrivain qui C. Arrighi introduit le mot «scapigliatura » en Italie pour traduire le terme de «bohème ». Ce sont les scapigliati qui «introduisent en Italie le naturalisme français ». Ce texte permet de comprendre quand, comment, et par qui est arrivé le naturalisme en Italie.

Le deuxième texte aborde quant à lui un courant littéraire typiquement italien appelé le «Verismo », nait en parallèle du naturalisme français. Ce courant possède ainsi des caractéristiques semblables au naturalisme. Deux auteurs sont importants, L. Capuana qui «analyse le malaise de la vie privée des bourgeois » à la manière de Zola et, G. Verga, qui créer un cycle romanesque, à la façon des Rougon Macquart de Zola, intitulé I Vinti. Ici, la rubrique «Trait d'union» explique les origines du « Verismo ». Voyons ce qu'il en est pour la rubrique « Liaison » d'Ecritures. En quelques mots, la rubrique «Liaisons » d'Ecritures (171) reprend ce qui a plus ou moins été dit dans la rubrique «Trait d'union » de Kaléidoscope. C'est grâce à la traduction (1875) de La Curée de Zola que les auteurs italiens Verga et Capuana initient le vérisme italien. (171 de Ecritures). L. Capuana est

Slavonic Pedagogical Studies Journal, ISSN 1339-866o, Volume 5 Issue 1, February 2016 
toujours le défenseur des théories de Zola alors que Verga est, tout comme Zola, le créateur d'un « cycle romanesque intitulé « I Vinti ».

Dans les manuels Beaubourg et Rive Gauche, nous retrouvons l'explication $\mathrm{du}$ 《 verismo » dans la rubrique « D'un pays à l'autre » et l'extrait est identique dans les deux manuels. Ici, la rubrique explique clairement ce qu'est le Vérisme : «dipingere dal vero », c'est-à-dire peindre à partir du réel, peindre la vie. L'explication s'appuie sur la définition du terme naturalisme selon E. de Goncourt, « étude d'après nature », définition qui vient du monde pictural. C'est donc en ce sens, nous dit-on, que «le vérisme est la traduction littérale de naturalisme ». Tout comme dans les autres manuels l'auteur considéré comme le fondateur de ce courant littéraire est L. Capuana. On nous donne par la suite un extrait de l'article du Corriere du 10 mars 1877 dans lequel L. Capuana montre tout son enthousiasme pour l'Assommoir de Zola qui réussit à « far penetrare il lettore nell'intimo spirito dei suoi personaggi », c'est-à-dire qu'il est capable de «faire pénétrer le lecteur dans la pensée intime de ses personnages ». Il ajoute que « la sensazione non rimane in lui al semplice stato di sensazione, ma s'innalza, si purifica, diventa sentimento, poesia. Poesia!" Ce qui signifie : «la sensation ne reste pas au simple état de sensation, mais elle s'élève, se purifie, elle devient sentiment, poésie. Poésie ! ».

\section{Le symbolisme de la poésie française et de la poésie italienne dans les manuels analysés}

Un autre point qui, dans Ecritures (199), prend une importance presque aussi grande que le romantisme, est celui sur le symbolisme et le décadentisme. Il se compose d'un petit extrait qui fait la liaison entre les poètes symbolistes français et les « crepuscolari » italiens (Écritures : 199). Cet extrait est identique dans Kaléidoscope (187) : La poésie symboliste tout comme la poésie des «crepuscolari » est une poésie «qui crée une atmosphère de magie grâce à la valeur musicale des mots ». Le couple Verlaine, Rimbaud influencera majoritairement « les décadents italiens et les crepuscolari ».

D'un côté, il y a l'aspect poétique avec Baudelaire et sa figure de style : la synesthésie qui est reprise par Montale et Pascoli. Les rubriques «Liaisons » (199 d'Ecritures) et «Trait d'union » (187 Kaléidoscope) mettent en relief ce parallélisme du renouveau symboliste de la poésie française et de la poésie italienne de Pascoli et des crépusculaires.

Dans Ecritures, nous avons à la suite quelques lignes sur le traducteur de Rimbaud, Zanzotto (202) : Le document se présente à nouveau sous la forme de deux colonnes. Cette fois-ci cette disposition ne suggère pas uniquement la comparaison, elle propose un parallèle entre le texte original, dans la colonne de gauche, extrait de la poésie de Rimbaud en langue française et, sa traduction par Zanzotto en langue italienne dans la colonne de droite. Ce lien est utile pour effectuer un travail de comparaison durant les cours de français langue non maternelle.

Puis, d'un autre côté, nous avons toute une page dédiée au décadentisme et au symbolisme en France et en Italie (203). Cette page reprend la rubrique 
«Liaisons » que nous avons vus lors de l'étude du romantisme. Que nous dit cette page?

Nous n'allons pas revenir sur le premier paragraphe intitulé « La bohème parisienne et la «scapigliatura» italienne puisque nous l'avons vu avec le «verismo » dans Kaléidoscope. Nous pouvons cependant souligner que l'extrait se positionne différemment d'un manuel à un autre.

Observons, en revanche, la deuxième partie intitulée « Le symbolisme et le décadentisme italien ». Dans un premier temps notons les auteurs français, Baudelaire, Verlaine, Rimbaud et Mallarmé, et les auteurs italiens, influencés par ce courant littéraire, G. D’Annunzio, G. Pascoli et A. Fogazzaro. Les thèmes communs à ces deux courants sont la musicalité, le symbole comme réalité, la synesthésie et le personnage de l'esthète image de «l'intellectuel décadent, qui se retire dans son refuge et vit en solitude dans l'impossibilité de s'adapter au monde ».

Nous sont ensuite présentés quelques auteurs du monde inspirés de et, inspirant ces mêmes courants : E. A. Poe aux USA ou encore O. Wilde en Angleterre.

Dans Kaléidoscope nous retrouvons ces trois mêmes aspects décadentisme, symbolisme et auteurs étrangers présentés de la manière suivante. Le lien entre symbolisme, décadentisme français et auteurs italiens se présente de la même manière que dans Ecritures. Nous avons d'abord quelques lignes sur les auteurs italiens G. D'Annunzio, G. Pascoli et A. Fogazzaro puis les thèmes clés du symbolisme et du décadentisme repris par ceux-ci. Kaléidoscope nous dévoile cependant que le «courant naturaliste et le courant décadent se développent parallèlement». Nous retrouvons une présentation similaire de la relation entre auteurs français et italiens dans les deux manuels, celle-ci insiste d'autant plus sur le courant italien et en dit très peu sur le courant français. Ecritures possède néanmoins un paragraphe supplémentaire intitulé «L'influence de Baudelaire, Rimbaud, Verlaine et Mallarmé de nos jours » qui explique le lien entre Zanzotto et ces poètes. On se rappelle que Zanzotto « traduit » Rimbaud ; on apprend maintenant que Zanzotto n'avait que 17 ans lorsqu'il découvre les œuvres de Rimbaud et traduit « immédiatement le Bateau ivre ». Puis, nous sommes renvoyés à l'extrait de la traduction des Chercheuses de Poux que nous avions vu auparavant.

Les manuels Beaubourg et Rive Gauche nous expliquent dans la rubrique «La vie des mots » ce qu'est le «Symbole » (174). Après avoir donné un aperçu du sens étymologique du mot «symbole », les auteurs des manuels Beaubourg et Rive Gauche expliquent le sens du mot symbole de nos jours, soit, une «association de deux réalités pour produire un nouveau signe ». Les symbolistes de l'époque, ont pris la « liberté » d'inventer de nouveaux signes en ayant recourt aux mythes et aux légendes si nécessaire, comme par exemple Orphée, symbole de la poésie.

Dans la rubrique suivante, « D'un siècle à l'autre » est mise en avant la continuité du mouvement au travers d'auteur-compositeurs français s'inspirant de Verlaine (175). Sont présentés ici des compositeurs tels que Fauré ou Debussy et des chanteurs tels que Ferré, Trenet ou Moustaki, qui

Slavonic Pedagogical Studies Journal, ISSN 1339-866o, Volume 5 Issue 1, February 2016 
ont repris les poésies de Verlaine dans leurs chansons. Par exemple «Le pauvre Gaspard » de Moustaki qui s'inspire des poèmes de Verlaine dans Sagesse III, 4. Ces deux manuels, Beaubourg et Rive Gauche, choisissent de parler de Dino Campana. Les œuvres de Campana ont reçues l'influence de Rimbaud. Par exemple, Le Bateau ivre de Rimbaud trouve certains échos dans les Canti orfici de Campana. «Les critiques les associaient au début du XXe siècle à cause de leur existence tourmentée », ils étaient en effet tous deux instables au niveau psychologique, et ils partageaient la même passion pour les voyages. Les titres des articles de l'époque sont claires à ce sujet: Campana est «l'ultimo bohémien d'Italia », on se souvient alors des termes «bohème » et «scapigliatura » que nous avons vu un peu plus haut. Les critiques choisissent volontairement de reprendre le terme français et non l'italien afin d'appuyer la comparaison avec Rimbaud. Ils l'appellent aussi le « Rimbaud della Romagna » car Campana est né dans la région italienne appelée la Romagne. Il est lui aussi considéré comme un « poète maudit».

\section{La représentation du XXe siècle dans les manuels analysés}

$\mathrm{Au} \mathrm{XXe}$ siècle, les liens littéraires entre la France et l'Italie sont moins nombreux par rapport à ceux entretenus à l'époque du Romantisme et du Vérisme. Cependant, le choix des auteurs français illustrant les manuels continue à dépendre des courants italiens. Les auteurs des manuels vont en effet souligner l'amitié de Apollinaire et Ungaretti (Sorbonne 1923), aborder les monologues intérieurs de Proust, de Colette et de Céline, le thème de la jalousie présent chez Proust et chez Svevo, appelé aussi « le Proust italien », et le roman policier qui nait aussi en Italie avec Scerbanenco ou Camillieri. On se souvient aussi du commissaire Montalbano téléfilm sur la RAI qui ressemble au commissaire Maigret. La philosophie est présente dans de nombreux thèmes que les extraits de textes littéraires soulignent: le mal, les passions, la morale... Les manuels présentent des philosophes célèbres allant de Sartre à Camus, de Simone De Beauvoir à Boris Vian. Les différents philosophes ont appartenu à des mouvements philosophiques différents : le positivisme, l'existentialisme, l'humanisme, le structuralisme, tous issus du XXe siècle.

L'un des plus gros impacts lors de ce siècle est, selon les auteurs des manuels, l'existentialisme de Sartre, Camus, ou Beauvoir. Celui-ci nait après la Seconde Guerre Mondiale, c'est « une philosophie de l'action, de l'effort, du combat, de la solidarité », une philosophie dans laquelle l'existence précède l'essence. Ce mouvement influence l'auteur italien Moravia. Le morcellement de la personnalité, le caractère illusoire de la réalité sont, en revanche, des thèmes plus proches de Beckett et de l'absurde, que nous retrouvons chez Pirandello.

Un autre mouvement clé du $\mathrm{XX}^{\circ}$ siècle : les OULIPO l'Ouvroir de Littérature Potentielle, un atelier où l'on œuvre à plusieurs, pour exploiter les potentialités de l'écriture. Quel est le lien entre les OULIPO et l'Italie ? Calvino, Italo Calvino, membre du groupe en 1980.

Une rubrique «Trait d'union » de Kaléidoscope est entièrement consacrée à Calvino. Nous apprenons que Calvino a vécu à Paris où il a connu le groupe 
Oulipo dont «il partageait l'idée de l'importance des contraintes dans l'œuvre littéraire, et l'applications de règles du jeu très strictes, le recours à des procédés combinatoires, le recours à des matériaux préexistants ». Il s'agit là des idées clés du mouvement de l'Oulipo. Le document présente un exemple, en langue maternelle italienne, de ce goût pour les règles : « Se una notte d'inverno un viaggiatore » roman paru en 1979.

Kaléidoscope propose comme activité à faire en classe ou seul pour approfondir, une comparaison entre ce début de roman avec celui de Perec exposé plus haut dans le manuel. Dans sa rubrique «Liaisons », le manuel Ecritures nous parle lui aussi de ce célèbre mouvement de l'Oulipo (377). Une image nous présente la couverture de la revue le Magazine littéraire ayant consacré un de ses numéros aux Oulipiens. Il s'agit du n 398 de mai 2001. Calvino, auteur italien, figurent parmi les auteurs qui sont présentés dans ce numéro, parmi les auteurs qui jouent avec la littérature. Le texte quant à lui, nous explique le lien entre Calvino et l'Oulipo. Calvino « devient membre du groupe en $1980 »$. Calvino explique les idées qui l'attirent au sein de ce mouvement littéraire, ce sont les mêmes idées que nous avons vu avec Kaléidoscope. Calvino a aussi traduit Queneau dans Contes italiens. En revanche, nous avons ici une présentation plus poussée de l'œuvre majeure de Calvino: Si par une nuit d'hiver un voyageur. Le roman s'inscrit dans le mouvement littéraire l'Oulipo, c'est une opération de réécriture (création d'œuvres nouvelles en utilisant des matériaux préexistants). Il se compose de dix débuts de romans, Calvino a recours aux procédés combinatoires, et respecte les contraintes des œuvres littéraires puisque dans ces dix débuts de roman, le noyau narratif et le schéma sont maintenus.

Nous retrouvons plus loin (379: Ecritures), un autre développement sur le mouvement de l'Oulipo. La première partie du document, présente une autre auteur français qui fait partie du groupe, M. Duchamp. A la fois "peintre, sculpteur et poète », il est l'auteur de la Joconde « affublée d'une moustache et d'une barbiche ».

Dans la deuxième partie du document, nous découvrons l'équivalent de l'Oulipo en Italie, l'Oplepo : Opificio di Lettura Potenziale. Ce mouvement regroupe écrivains et poètes, Sanguinetti, Umberto Eco, mathématiciens Odifreddi, savants, chercheurs et informaticiens.

Dans Beaubourg et Rive Gauche, une explication du mouvement de l'Oulipo, apparaît dans la rubrique «Au premier plan». C'est le mathématicien humaniste $\mathrm{F}$. Le Lionais qui est à l'origine de ce courant littéraire. De nouveau nous retrouvons une référence à Calvino, quoique minime. On nous confirme uniquement le «principe que toute poétique obéit à des règles ». Leur but est « d'inventer de nouvelles structures » tout en ayant de « grandes responsabilités ludiques».

Sur le plan littéraire, les auteurs des manuels de «quinta», au centre de notre recherche, semblent orienter leurs choix des thématiques et des auteurs à étudiés, en fonction des rapports ou des liens qu'ils entretiennent avec l'Italie. Ce rapprochement se note surtout dans les rubriques « Trait d'union » de Kaléidoscope, «Liaison» d'Ecritures et « La vie des mots »

Slavonic Pedagogical Studies Journal, ISSN 1339-866o, Volume 5 Issue 1, February 2016 
ou «D'un pays à l'autre » de Beaubourg et Rive gauche. Les manuels abordent ainsi la littérature française sous l'angle de la littérature italienne.

Kaléidoscope propose surtout des comparaisons à effectuer en classe. Il s'agit notamment de textes en langue italienne à comparer avec des textes en langue française. Le lecteur doit obligatoirement connaitre l'italien pour affronter ces activités.

Selon nous, Ecritures est le plus complet de tous les manuels. Il explique sans ambiguïté chacune des thématiques abordées ainsi que les liens qui existent entre France et Italie. L'apprenant est mieux guidé et orienté et peut ainsi mieux saisir l'emploi de tel ou tel argument, ou encore pourquoi tel auteur ou tel extrait peut- être comparé à tel autre. Il s'agit de réels approfondissements.

Beaubourg et Rive Gauche sont plus théoriques. L'explication d'un mot ou d'un auteur devient «illustration» par rapport au contenu des extraits littéraires. En effet, lors des développements de la vie d'un auteur ou autre, on note la présence du symbole suivant. C'est un triangle rouge à l'envers qui comme un astérisque renvoie à l'explication ou à l'approfondissement d'un mot, d'un mouvement, d'un auteur ou d'un lien franco-italien. C'est une façon d'illustrer ce que les chapitres ou leçon développent. Voilà pourquoi nous préférons employer le mot « illustration » pour parler de ces rubriques qui sont bien un exemple de ce que les auteurs des manuels abordent auparavant.

\section{La culture politico-historique dans les manuels analysés}

Chacun des manuels étudiés, aborde la culture politico-historique de la France et de l'Italie. Dans Ecritures, nous la retrouvons dans les onglets «Histoire et société », dans Rive Gauche et dans Beaubourg, elle est abordée dans «Un peu d'histoire ».

Kaléidoscope, quant à lui, y dédie plusieurs unités : l'unité 1 du module 1 sur Napoléon et la révolution de 48, l'unité 1 du module 2 sur Napoléon III et la troisième République, l'unité 1 du module 3 sur les deux grandes Guerres Mondiales (14 et 45) et l'unité 1 du module 4 sur la quatrième et la cinquième République, ainsi que sur l'Empire coloniale.

Partons des extraits de «Esprit du siècle » de Kaléidoscope, de «Esprit du siècle » (216), « Transgressions et engagement »d'Ecritures ainsi que des tables des matières que nous avons vu lors de l'étude de la mésostructure des manuels de «quinta » afin de définir les différents axes d'études au niveau historique dans ces mêmes manuels. Commençons par le XIXe siècle.

A gauche nous avons l'extrait d'Ecritures et à droite celui de Kaléidoscope. Le contenu textuel est identique, seule la présentation change. Nous retrouvons la forme géométrique du schéma chez Kaléidoscope, ici il s'agit d'un cercle. Une seule couleur pour les caractères, le noir, seul le titre est en gras pour se différencier du corps du paragraphe. Nous avons ici une sensation de clarté. Le simple fait d'avoir changé la couleur du titre par rapport au corps du texte donne une touche supplémentaire à Ecritures. Les illustrations rendent la présentation d'Ecritures beaucoup plus attrayante. 
En comparant ces documents et les tables des matières, nous remarquons que les thèmes sont plus ou moins identiques d'un manuel à un autre. En effet, le XIXe siècle se caractérise par :
a) deux empires (Napoléon I et Napoléon III),
b) trois monarchies (Louis XVIII, Charles X, Louis Philippe)
c) deux républiques (1848-1851 et 1871)
d) trois révolutions $(1830,1848$ et 1870$)$
e) La naissance des parties politiques

Effectuons le même travail pour le XXe siècle.

Comme pour le XIXe siècle, à gauche nous avons un extrait d'Ecritures et à droite de Kaléidoscope. Le contenu textuel est ici aussi identique, seule la présentation change. Nous retrouvons à nouveau une forme géométrique de type schéma chez Kaléidoscope, dans ce cas il s'agit d'un rectangle, monté sur un triangle. Une seule couleur pour les caractères, le noir, seul le titre est en gras pour se différencier du corps du paragraphe. Nous retrouvons la même recherche de simplicité. Dans Ecritures le fait d'avoir changé la couleur du titre par rapport au corps du texte donne une touche supplémentaire. Les illustrations rendent ici encore, la présentation d'Ecritures très attrayante.

En comparant ces documents et les tables des matières, nous remarquons que les thèmes sont plus ou moins identiques d'un manuel à un autre. En effet, le XXe siècle se distingue par :

a) Les deux grandes Guerres (14-18 et 40-45)

b) La décolonisation

c) La francophonie

d) L'Europe

Nous classons aussi sous la rubrique culture politico-historique les différents personnages historiques tel que Napoléon Ier, les images qui dépeignent les personnages historiques et/ ou politique (portraits et autres), les images qui décrivent les guerres..., le roman historique et les hommes politiques des différentes Républiques.; Sont catalogués comme hommes politiques les personnages tels que Jacques Chirac et François Mitterrand (312-Kaléidoscope) ou bien Le général De Gaulle (304 -Ecritures).

Nous nous demandons à présent si les thèmes historiques abordés sont présentés de la même manière aux étudiants italiens de français langue non maternelle, d'un manuel à un autre. Quelles sont les parties historiques qui sont mises en valeur? Pourquoi? Quels personnages historiques apparaissent comme plus importants?

En dehors des onglets historiques tels que «Un peu d'histoire» de Rive Gauche ou tel que «Histoire et société »d'Ecritures, les manuels de « quinta » s'attardent tout particulièrement sur le personnage historique de Napoléon Ier. Dans Beaubourg et Rive Gauche, une rubrique «Personnage » est entièrement consacrée à « Napoléon en 1815 ».

Les auteurs du manuel évoquent en premier lieu la célèbre évasion de l'île d'Elbe et l'appel nationaliste de Napoléon aux soldats français, il les

Slavonic Pedagogical Studies Journal, ISSN 1339-8660, Volume 5 Issue 1, February 2016 
exhorte « à l'orgueil national et à la revanche ». C'est un des points clé du personnage qui a provoqué l'admiration à son arrivée en Italie.

Quant à Kaléidoscope il consacre toute l'étape 3 du module 1 à « La légende de Napoléon ». Ecritures reprend cette même légende dans une double page. Le récit se présente comme un article de journal, en deux colonnes, permettant ainsi de concentrer le texte sur une même page. Seuls les titres sont en caractères gras, pour se différencier du reste du texte. Selon nous, cette présentation est terne, elle ne donne pas envie de lire ce qu'il y a à l'intérieur. Nous avons réellement l'impression de nous trouver face à un article de journal peu attrayant.

Les contenus textuels d'Ecritures (14-15) sont identiques à ceux de Kaléidoscope. La mise en forme change cependant. Les textes sont ici plus aérés. Le simple fait d'avoir énuméré les paragraphes avec la couleur rouge, d'avoir mis des points rouges au lieu des tirets dans la première partie intitulée "La légende officielle », et d'avoir illustré le propos avec des images, rendent le récit beaucoup plus attrayant dans le manuel Ecritures.

Du point de vue du contenu. Il nous est dit que «Chateaubriand, (...), fait naître Napoléon un an plus tôt quand la Corse n'était pas française pour insister sur ses origines étrangères ». De la même manière, Kaléidoscope et Ecritures soulignent le fait que Napoléon Bonaparte est né en Corse et que «Gènes venait de céder un an plus tôt l'île à la France » (Écritures : 12). Ainsi les auteurs de ces deux manuels insistent sur l'origine et la culture italienne dans laquelle a bercé Napoléon instaurant par là un premier lien entre Napoléon I ${ }^{\text {er }}$ et l'Italie.

Par la suite nous apprenons que Napoléon «se voit confier le commandement de l'armée d'Italie » (Écritures : 12), comme pour mettre en relief cet épisode dans la vie de Bonaparte, les auteurs des manuels ont ici volontairement adopté le caractère gras. Il entre dans Milan en 1796 et instaure ultérieurement plusieurs républiques (la République romaine, la République de Vénétie...etc.) C'est avec la victoire de Marengo qu'il devient Roi d'Italie. Il y a donc un lien profond entre ce personnage, son histoire et l'Italie.

En outre, à l'aide de leur rubrique «Liaisons » pour Ecritures, et « Trait d'union », pour Kaléidoscope, les auteurs attirent l'attention sur Napoléon et les campagnes d'Italie.

Nous observons un paragraphe supplémentaire dans l'encadré d'Ecritures : «Napoléon et les écrivains européens ». Nous y retrouvons quelques écrivains inspirés par Napoléon. Trois auteurs italiens sont cités, Alfieri, Leopardi et Manzoni, en revanche aucun auteur français n'apparaît ici. Nous supposons alors que nous allons en trouver dans la suite des manuels. Puis, suivent quelques lignes sur l'Ode de Manzoni pour Napoléon qui s'intitule : Il Cinque maggio. Le poème est mentionné chez Ecritures, il est entièrement retranscrit dans Kaléidoscope. Quelques lignes d'explication sont données en français avant le texte en langue originale italienne.

Fermons la parenthèse du cinq mai et revenons aux rubriques consacrées aux campagnes d'Italie et à leurs différences. La photo de Napoléon rend le manuel Ecritures plus attrayant que son ainé. 
Le contenu textuel des deux manuels est quasi identique et commence ainsi : «Lorsque Bonaparte a commencé sa campagne d'Italie, il a bénéficié du soutien des patriotes italiens admirateurs de la Révolution française ». Les italiens ont majoritairement reçu la venue de Bonaparte avec enthousiasme, «si bien que le jeune général a été accueilli en libérateur ». Notons à nouveau la frugalité de Kaléidoscope.

Les deux manuels soulignent les premiers manquements et les premières désillusions :

a) Bonaparte «sacrifie » la République de Vénétie aux Autrichiens (Traité de Campoformio).

b) La France par son besoin d'argent «impose lourdement les Italiens ».

c) Les « graves » pillages de Bonaparte.

Seul Kaléidoscope propose un extrait de Ugo Foscolo, Ultime lettere di Iacopo Ortis (1802), pour démontrer « le désespoir des patriotes italiens ». Ecritures se contente de nommer quelques auteurs qui traitent le sujet: Manzoni, Alfieri et Leopardi.

Dans ce nouveau «Trait d'union » nous repérons le même procédé que pour l'Ode du cinq mai, quelques lignes d'explication en français précèdent le texte écrit en langue maternelle italienne.

Arrivé à ce point, il nous semble que les auteurs des manuels de «quinta » racontent l'histoire de la France pour mieux raconter l'histoire de l'Italie. En effet, durant le second Empire et Napoléon III, la France joue un rôle important dans l'unité italienne, nous comprenons ainsi la place consacrée à cette période dans les manuels. Ce rôle est expliqué de la même manière dans la partie « Liaisons » du manuel Ecritures (127) :

que dans la rubrique «Trait d'union » du manuel Kaléidoscope. Les extraits des deux manuels commencent ainsi :

« La France est intervenue en Italie en tant que puissance catholique contre la jeune république mazzinienne » (Rome le 9 février 1849). Ils soulignent donc tous deux l'entrée de la France sur le territoire italien.

Par la suite, les auteurs mettent en avant une défaite française : « le 30 avril, les forces de Garibaldi venues de toutes l'Italie infligent une première défaite aux français à Velletri ». Bien que cette défaite n'arrête pas les forces françaises qui battent Garibaldi à Rome.

« La politique de Napoléon III pourrait apparaitre confuse, car s'il poursuit les libéraux en France, il les appuie à l'étranger » en appuyant Cavour. A posteriori, le plan de Napoléon III apparaît plus clair, il voulait obtenir une Italie modérée et neutraliser les extrémistes.

De la troisième République, ressortent de nombreuses idées phares du libéralisme : liberté de la presse, désobéissance militaire, droit de se réunir et de former des syndicats.... Ce sont des idées que l'Italie reprendra lors du Risorgimento.

Sur quels arguments les auteurs font-ils une impasse ? Pourquoi ?

Dans le manuel Ecritures, Huit pages (220-227) reprennent la première et la deuxième Guerre Mondiale. Elles ont toutes deux aussi bien marqué l'Histoire qu'inspiré le cinéma et la littérature française (par exemple pour le

Slavonic Pedagogical Studies Journal, ISSN 1339-866o, Volume 5 Issue 1, February 2016 
cinéma, la Grande Vadrouille de G.Oury (1966), la comédie fondée sur le mythe de la résistance et pour la littérature Saint Exupéry - Pilote de guerre- ou encore Sartre - Le Mur.), comme italienne (avec Carlo Levi Cristo si è fermato a Ebol i- avec Primo Levi - Se questo è un uomo - ou bien Calvino - Il sentiero dei nidi di ragno). Ecritures met en avant le lien entre histoire, littérature et cinéma.

Le document traite de la guerre, de l'occupation et de la libération pour mieux parler du lien entre histoire et cinéma et leur rapport avec l'Italie, qui reste ici encore, le point fondamental du manuel.

Le rôle de l'Italie dans la Deuxième Guerre mondiale n'est pas des moindres, notamment avec Mussolini et sa dictature fasciste. Pourtant la rubrique aborde à peine la présence de l'Italie en guerre : «l'Italie déclare la guerre à la France » pour « récupérer le Comté de Nice, la Corse et la Tunisie ». Les auteurs d'Ecritures, font l'impasse sur les relations francoitaliennes pendant la deuxième Guerre Mondiale, alors qu'entre autres, les deux pays se sont affrontés lors de plusieurs batailles. Kaléidoscope en revanche souligne «l'occupation italienne de Nice», période d'environ deux ans, durant laquelle les italiens ont eu à nouveau le contrôle sur la ville, ainsi que sur une zone plus large de l'actuel sud-est français.

Le document débute par une brève introduction sur le positionnement de l'Italie durant la première puis la Seconde Guerre Mondiale. Il explique ainsi l'alliance de l' "Italie fasciste » avec l'Allemagne d'Hitler, donnant notamment lieu à l'occupation italienne de Nice, autrefois ville italienne. Pour illustrer la réalité d'une telle occupation, les auteurs de Kaléidoscope ont choisi de présenter un texte autobiographique relatant une conséquence de l'occupation : le marché noir. Les manuels de Beaubourg et Rive gauche font eux une parenthèse sur le régime de Vichy.

Dans cet encadré, nous vivons l'arrivée au pouvoir du Général Pétain, et l'instauration du régime autoritaire de Vichy, qui marquera le début de la collaboration de l'Etat français avec l'Allemagne nazie.

Seuls Beaubourg et Rive Gauche proposent le témoignage d'un déporté avec un extrait du texte Souvenir de la Maison des Morts du résistant R.P. Riquet.

Les quatre manuels que nous analysons ne parlent que brièvement de la libération. Nous avons cependant trouvé intéressant le passage intitulé « La libération selon Simone de Beauvoir » dans Beaubourg et Rive Gauche.

En réalité, les autres manuels proposent eux aussi des textes littéraires sur le sujet, mais ces derniers font partie intégrante de l'unité, c'est à travers eux que nous découvrons les thèmes importants de l'histoire. Cependant pourquoi faire particulièrement ressortir certains extraits avec des encadrés en dehors du corps même de l'unité ? Après relecture des manuels, nous constatons que ces sujets n'ont pas été traités spécifiquement dans l'unité au travers des extraits littéraires intégrés dans l'unité. C'est ainsi l'occasion de le faire et d'apporter un éclaircissement lorsque les manuels nomment la deuxième Guerre Mondial dans la rubrique «Un peu d'histoire ».

Après les deux grandes Guerres, les manuels traitent rapidement les IVe et Ve Républiques, puis ils couchent une liste des présidents de la $V^{\circ}$ République : Générale de Gaulle (1958 - 1969), Pompidou (1969 - 1974), 
Giscard d'Estaing (1974 - 1981), puis Mitterrand (1981 - 1995), Chirac $(1995$ - 2007) et Sarkozy $(2007$ - 2012). La fin du mandat de ce dernier n'est pas insérée dans les manuels car il est encore au pouvoir lors de leur parution.

\section{Les écrivains et la politique dans les manuels analysés}

Kaléidoscope dédie une page aux écrivains et à leur engagement politique ; Classé sous la catégorie «contexte» le document se présente sous forme journalistique, en deux colonnes, certainement pour une question d'espace. Il comporte trois grands paragraphes. Seul le titre est de couleur verte, le reste est en noir. Le nom des auteurs cités est en caractères gras pour les détacher du corps du texte et «taper dans l'œil » des étudiants italiens. Une photo de Victor Hugo en noir et blanc illustre l'article. Dans Ecritures c'est une double page qui présente cette thématique sous la rubrique « panorama littéraire et culturel »: Les grands paragraphes sont au nombre de trois, les noms des auteurs sont toujours en gras. Les photos illustratives changent et augmentent au nombre de trois. Hormis les sept premières lignes, qui sont unique à Ecritures, la suite du texte est identique dans les deux manuels. Dans l'introduction du texte d'Ecritures, nous apprenons que souvent les auteurs romantiques se sentent investis d'une mission, celle de guider l'humanité. Les trois auteurs cités sont Lamartine, Hugo et Vigny. Lamartine met « son talent littéraire au service des idées philosophiques et morale afin de contribuer à l'édification du peuple». Hugo est un «prophète qui éclaire le peuple, et le conduit à Dieu ». Le lien entre la littérature et l'histoire est ici fondamental, il permet aux auteurs de faire passer leurs idées à travers leurs écrits et de transmettre une image de leur société.

Deux documents sont présents dans Ecritures. Le premier est le discours de Lamartine alors «membre du gouvernement provisoire de la deuxième République ", publié dans Le Moniteur universel du 7 octobre 1848. Il y défend l'élection du président par le suffrage universel. Le deuxième est extrait des Contemplations de V. Hugo, livre III, et dénonce le travail des enfants.

Beaubourg et Rive Gauche ne développent pas la thématique des écrivains et de leurs idées politiques.

\section{Bilan de la microstructure et de la culture dans les manuels de « quinta »}

Il est clair qu'à la suite des données que nous venons d'étudier, les différents domaines de la langue cible qui sont majoritairement traités ne sont ni la grammaire-conjugaison, ni le vocabulaire, et ni la phonétiqueintonation. Ces points qui étaient fondamentaux dans les manuels de la première année du lycée sont totalement absents des manuels de « quinta ». $\mathrm{Au}$ niveau des compétences linguistiques, les objectifs des manuels de « quinta » semblent « muets » sur le sujet. Notre analyse nous permet de confirmer la recherche de deux compétences : la compréhension et la production écrite. L'oral est complétement absent. 
Les manuels suivent une progression chronologique, qui suit la chronologie historique des mouvements littéraires, philosophiques et culturels.

L'organisation cognitive des savoirs s'effectue selon un catalogue préétabli d'extraits de textes littéraires.

La méthode qui prévaut dans les manuels étudiés, est une méthode traditionnelle, c'est-à-dire l'enseignement d'une langue centré sur l'écrit et l'importance de l'enseignement-apprentissage de la littérature.

Quelle conception de la culture française est apportée par les manuels scolaires aux étudiants italiens?

Les auteurs ont choisi de faire une comparaison ou un rapprochement régulier entre culture française et culture italienne. Ceci permet aux apprenants de découvrir une nouvelle culture, la culture de la langue cible, le français, mais aussi de mieux connaitre leur propre culture. Les manuels sont-ils des manuels de français ou bien des manuels d'italien ? Selon nous, ils retracent la culture française pour mieux parler de la culture italienne. Ils insistent sur certains aspects du passé, comme la présence et le rôle de la France dans l'unification de l'Italie, et en minorent d'autres comme la présence et le rôle de l'Italie dans la Deuxième Guerre Mondiale. Ceci, toujours selon nous, dans le but de faire ressortir les éléments positifs de l'histoire, de la littérature et de la culture italienne face à l'histoire, la littérature et la culture française.

Nous avons omis volontairement l'analyse du premier volume de Rive Gauche. Seuls ses outils pour lire, analyser et écrire servent réellement en classe de «quinta » car la période historique, du Moyen Age au XVIIIe siècle, n'est pas dans le programme didactique des classes de lycée en Italie. Entre autre, dans les manuels de «quinta », la culture est séparée de la linguistique. Seul l'aspect culturel est présent. Il s'agit d'une culture cultivée. Aucun signe de quotidienneté n'apparaît, aucun intérêt proche du commun des adolescents. Tout ce qui est à la base du manuel de prima (grammaire, phonétique...) a disparue dans les manuels de quinta. Cette analyse confirme donc notre hypothèse de la présence d'un fossé didactique dans les manuels de français non maternel entre la première année et la dernière année du lycée italien.

En réalité, nous avons l'impression de retrouver à travers ces manuels de « quinta », l'un des buts que Maurer (2011 : 49) propose dans son premier scénario pour la formation des professeurs. Ce but, est de dirigé autant les apprenants que les enseignants: «mettre en évidence les différences d'ordre culturels d'un pays à un autre. " Tout comme Maurer, nous nous demandons l'intérêt formatif d'une telle démarche. En effet, dans notre cas, les auteurs de manuels italiens ne disent pas tout, ils essaient de mettre en relief les aspects positifs de la culture italienne bien souvent au détriment de la culture française. Par exemple, évoquons la manière dont est présentée la naissance du romantisme avec Madame de Staël. Les auteurs semblent prendre plaisir à préciser qu'il ne nait pas exactement en France, mais en Allemagne. Car, à l'époque, Madame de Staël était exilée en Allemagne. «C'est à une femme que l'on doit le premier essai sur le romantisme en français: Germaine de Staël » nous dit-on dans Ecritures, pour mieux souligner : « le romantisme n’est donc pas né en France (...). Il est d'abord 
apparu en Allemagne et en Angleterre. » C'est une manière de démentir ceux qui affirment que le romantisme est avant tout français. Cette analyse culturelle sur les manuels de «prima» et les manuels de «quinta » a confirmé ce que les programmes scolaires des lycées italiens, vus dans notre première partie (voir grand 1), nous laissaient entendre, c'est-à-dire qu'il existe un fossé didactique, linguistique et culturel entre les deux classes.

$\mathrm{Au}$ fond, cette manière d'enseigner est un aperçu de ce que l'Europe attend des pays de sa communauté : un enseignement qui prévoie de donner des bases minimes d'une langue les premières années afin que l'apprenant puisse se débrouiller dans une situation de communication quelconque. Puis un « cours d'introduction aux grandes œuvres littéraires/philosophiques/de philosophie politique/des sciences sociales ... de la production (surtout) européenne, en version originale et en traduction (cycles secondaires) » (proposé par le guide des curriculums - 2010 : 41) à la fin des années lycées, pour souligner les différences culturelles des pays européens. L'aspect linguistique disparait peu à peu pour laisser place à une «didactique du plurilinguisme ». Est-ce réellement une meilleure didactique ? Si oui, alors pourquoi avons-nous rencontré bon nombre d'élèves qui à la fin des années de lycée ne savent pas écrire une phrase correcte en français ? Et pourquoi les établissements scolaires sont toujours plus nombreux à demander aux apprenants l'achat d'une grammaire si la grammaire ne fait pas partie du programme en classe de « quinta »?

\section{Bibliographic references}

BONINI, G. F. - JAMET M. C. - BACHAS P. - VICARI E. V. 2009. Ecritures, Anthologie litteraire en langue française, Valmartina.

BONINI, G. F. - JAMET M. C. 2010. Kaleidoscope. Volume C. Litterature et civilisation, de Napoleon a l'an 2000, Valmartina.

BERTINI, M. - ACCORNERO, S. - BONGIOVANNI, C. - GIACHINO, L. 2002. Beaubourg. Auteurs, textes, genres de la litterature française Einaudi scuola.

BERTINI, M. - ACCORNERO, S. - BONGIOVANNI, C. - GIACHINO, L. 2004. Rive gauche. Auteurs, textes, genres de la litterature française Einaudi scuola.

MAURER B. 2011. Enseignement des langues et construction européenne. Le plurilinguisme, nouvelle idéologie dominante, Edition des Archives Contemporaines. Paris.

Cynthia Etheves

Sciences of the Language

Univesity Paul Valéry 3

Route de Mende, 34100 Montpellier

France

cynthia.etheves@laposte.net 